INT. J. REMOTE SENSING, 1997, VOL. 18, NO. 16, 3395-3408

\title{
An investigation of remotely-sensed soil depth in the optical region
}

\author{
S. LIANG \\ Laboratory for Global Remote Sensing Studies, Department of Geography, \\ University of Maryland, College Park, Maryland 20742, U.S.A.
}

(Received 7 May 1996; in final form 8 January 1997)

\begin{abstract}
The remotely-sensed soil depth in the visible and near-infrared (near-IR) spectral region is investigated by using a numerical radiative transfer model of the coupled atmosphere and soil media. The sensible depth is determined by examining the downward hemispheric transmittance profile, hemispherical reflectance, and bidirectional reflectance with different solar zenith angles at different wavelengths. The particle size distributions and particle shapes are also evaluated. Under an ordinary condition, the sensible optical depth is about 3, which corresponds to geometric depth of 4-5 times the particle effective radius.
\end{abstract}

\section{Introduction}

Radiative transfer theory has been widely used for modelling bidirectional reflectance of different terrestrial objects that usually reflect very anisotropically. Compared to vegetation canopy, soil radiative transfer has been poorly investigated. The Hapke model (Hapke 1981, 1993) that was originally developed for reflectance of planetary surfaces has recently been used for soil inversion experiments (Pinty et al. 1989, Jacquemoud et al. 1992, Privette et al. 1995). Since the Hapke model assumes isotropic multiple scattering that is probably suitable for planetary surfaces, it is not very accurate for soils, since soils usually have much higher single-scattering albedo and larger asymmetry parameter (Mishchenko 1994, Liang and Townshend 1996a,b). Generally speaking, the Hapke model overestimates reflectances in the forward scattering direction and underestimates reflectances in the backscattering directions for the Earth's soil surface. Several improved versions have been published (Ahmad and Deering 1993, Liang and Townshend 1996a). A new soil radiative transfer model based on the fourstream approximation for multiple scattering has also been developed (Liang and Townshend 1996b). The hot spot effects using coherent backscattering theory have been calculated for small particles that do not have well-defined shadows (Liang and Mishchenko 1997). In spite of these eff orts, many fundamental problems have not been solved. For example, is the far-field approximation valid for the soil medium where particles are highly packed? How can we link the optical parameters required for radiative transfer calculations with soil inherent parameters, such as structure, organic matter, moisture and so on (Irons et al. 1989)? To answer those questions, comprehensive theoretical investigations are required. Answering these questions may rely on the estimation of the sensible soil depth in the optical region. If the sensible soil depth is large, the verticle profiles of soil moisture and other biophysical or geophysical variables may need to be considered. If small soil depths are detected, other factors such as soil 
surface roughness may become more important. Therefore, estimating the remotelysensed soil depth may affect the research directions on soil bidirectional reflectance modelling to some extent.

It has been observed that large penetration depths through particulate medium such as soils, snows and ices may take place in the microwave region, ranging from a few millimetres to dozens of metres (Ulaby et al. 1982). The penetration depth $\delta_{p}$ is defined as

$$
\int_{0}^{\delta_{p}} k_{e}(z) \mathrm{d} z=1
$$

where $k_{e}(z)$ is the extinction coefficient of the medium. In the visible and nearinfrared spectral regions, the penetration depth is usually much smaller. To estimate the soil depth in the optical region, we shall examine the downward transmittance profile and the dependence of upward radiance/reflectance on the depth using a numerical code DISORT, which is based on the discrete ordinate algorithm (Stamnes et al. 1988). This code has been well tested and used for many different applications (Tsay et al. 1989, Tsay and Stamnes 1992, Lindner 1988, Lummerzheim et al. 1989, Liang and Strahler 1994, Liang and Townshend 1996a,b). The atmosphere and soil are considered as a coupled medium at two different layers, each with different optical properties. The major advantage of coupling the atmosphere and the soil is to account for their interactions more effectively.

\section{Theoretical methods}

Both atmosphere and soil are treated as a particulate medium whose optical properties are calculated based on individual particles. In this study, particles are assumed spherical or ellipsoidal. Mie theory (Bohren and Huffman 1983) and the $T$-matrix method (Mishchenko 1993) are used for spherical and ellipsoidal particles, respectively. The soil particle distribution can be represented by different functions, such as lognormal (Buchan 1989) and Rosin distribution (Dapples 1975). For simplicity, the gamma formula is used to characterize the soil particle size distribution (Hansen and Travis 1974) in this study:

$$
n(r)=C r^{\left(1-3 v_{\text {eff }}\right) / v_{\text {eff }}} \exp \left[-r /\left(r_{\text {eff }} v_{\text {eff }}\right)\right],
$$

where $C$ is the normalization constant. The effective equal-volume-sphere radius $r$ eff and the effective variance $v_{\text {eff }}$ are expressed

$$
\begin{aligned}
r_{\mathrm{eff}} & =\int_{r_{1}}^{r_{2}} r \pi r^{2} n(r) d r / \int_{r_{1}}^{r_{2}} \pi r^{2} n(r) d r \\
v_{\mathrm{eff}} & =\int_{r_{1}}^{r_{2}}\left(r-r_{\mathrm{eff}}\right)^{2} \pi r^{2} n(r) d r / r_{\mathrm{eff}}^{2} \int_{r_{1}}^{r_{2}} \pi r^{2} n(r) d r
\end{aligned}
$$

where the particle radius lies in the range $\left(r_{1}, r_{2}\right)$. 
The equation of an ellipsoidal surface can be expressed in the spherical coordinate system

$$
r(\theta, \phi)=a\left(\sin ^{2} \theta+\mathrm{d}^{2} \cos ^{2} \theta\right)^{-1 / 2}
$$

where $d=b / a$, the ratio of the semi-axes of the ellipsoids. We can describe the particle shape ranging from needles $(d \ll 1)$ to discs $(d \gg 1)$ by varying the parameter $d$. If $d=$ 1, particles are spherical, and thus Mie theory can be used for calculating those optical properties. For general ellipsoidal particles, the $T$-matrix approach (Mishchenko 1993) is used in this study.

The optical depth $\tau$ is more frequently used than the real geometric depth $z$ in radiative transfer calculations. They can be converted through the following relation:

$$
\tau(z)=\int N(t)\left\langle C_{\text {ext }}(t)\right\rangle d t \approx\langle N\rangle\left\langle C_{\text {ext }}\right\rangle z
$$

where $\langle N\rangle$ is the average number density of particles and can be determined based on the proportions of the particle volumes occupied in the soil $(f)$

$$
\langle N\rangle=\frac{f}{\frac{4}{3} \pi r_{\mathrm{eff}}^{3}} .
$$

$\left\langle C_{\text {ext }}\right\rangle$ is the extinction cross-section averaged over size, shape, refractive index and orientation distributions. For spherical particles, the extinction cross-section can be easily calculated using Mie theory:

$$
\left\langle C_{\text {ext }}\right\rangle=C_{\text {ext }}=\frac{2 \pi}{k^{2}} \operatorname{Re} \sum_{n=1}^{\infty}(2 n+1)\left(a_{n}+b_{n}\right)
$$

where $k=2 \pi / \lambda$ and $\lambda$ is the wavelength, $a_{n}$ and $b_{n}$ are Mie coefficients (Bohren and Huffman 1983). For ellipsoidal particles, the corresponding formula given by the $T$ matrix method is (Mishchenko 1993)

$$
\left\langle C_{\text {ext }}\right\rangle=-\frac{2 \pi}{k^{2}} \operatorname{Re} \int_{n=1} \sum_{n^{n}=-1}^{n}\left[T_{m n m n}^{11}(A)+T_{m n m n}^{22}(A)\right]
$$

where $T^{i j}$-matrices are the essential parts of the $T$-matrix algorithm. Given the particle size distribution, complex refractive index and wavelength, Mie theory and the $T$-matrix algorithm provide not only the extinction cross-section, but also singlescattering albedo and phase function, which are necessary for radiative transfer calculations.

For a plane-parallel homogeneous semi-infinite medium in the absence of polarization, the radiative transfer equation for scattering radiance $I(\tau, \Omega)$ and appropriate boundary conditions can be written as (Lenoble 1985)

$$
\begin{aligned}
\mu \frac{\partial I(\tau, \Omega)}{\partial \tau}= & I(\tau, \Omega)-\frac{\omega(\tau)}{4 \pi} \int_{4 \pi} P\left(\tau, \Omega^{\prime}, \Omega\right) I\left(\tau, \Omega^{\prime}\right) \mathrm{d} \Omega^{\prime} \\
& +\frac{\omega(\tau)}{4} F_{0} P\left(\tau, \Omega_{0}, \Omega\right) \exp \left(-\frac{\tau}{\left|\mu_{0}\right|}\right)
\end{aligned}
$$




$$
\begin{gathered}
I(0, \Omega)=0 \\
\lim _{\tau \rightarrow \infty} I(\tau, \Omega)=0
\end{gathered}
$$

which imply that the coupled medium is illuminated by a parallel beam in the direction $\left(\Omega_{0}\right)$ with net flux $i_{0}=\pi F_{0}$ on the upper boundary and the upwelling radiance can be neglected for deep layers of the soil at the lower boundary. $\mu$ is the cosine of the viewing zenith angle and $\mu_{0}$ is the cosine of the solar zenith angle. Since the direct illumination has been explicitly included in the radiative transfer equation, the boundary condition at the top of the atmosphere is for the diffuse illumination only.

In equation (a), $\omega(\tau)$ is the single-scattering albedo, $P\left(\tau, \Omega^{\prime}, \Omega\right)$ is the phase function, and $I(\tau, \Omega)$ is scattered radiance in the direction $\Omega$ at optical depth $\tau$. $\Omega$ consists of an azimuthal angle $\phi$ and a zenith angle $\theta=\cos ^{-1}(\mu)$ which is positive for the upwelling directions and negative for the downward directions.

The radiative transfer equation can be solved through different algorithms (Lenoble 1985). The numerical code DISORT is used in this study, which is based on the discrete ordinate algorithm (Stamnes et al. 1988, 1996). The major ideas of this algorithm are outlined here. The azimuthal dependence of radiance is firstly decomposed in a Fourier cosine series:

$$
I(\tau, \mu, \phi)=\sum_{m=0}^{2 N^{-1}} I^{m}(\tau, \mu) \cos m\left(\phi_{0}-\phi\right)
$$

With this transformation, the original radiative transfer equation is converted to $2 \mathrm{~N}$ independent equations:

$$
\mu \frac{\partial I^{m}(\tau, \mu)}{\partial \tau}=I^{m}(\tau, \mu)-J^{m}(\tau, \mu) \quad m=0,1,2, \ldots, 2 N-1
$$

For simplicity, other terms in the right side of equation (9) have been denoted by $J(\tau, \Omega)$, which is often called the source function. The next step is to replace the integral by a quadrature sum and thus the above radiative transfer equation becomes a system of coupled differential equations:

$$
\mu_{i} \frac{\partial I^{m}\left(\tau, \mu_{i}\right)}{\partial \tau}=I^{m}\left(\tau, \mu_{i}\right)-J^{m}\left(\tau, \mu_{i}\right) \quad i= \pm 1, \ldots, \pm N
$$

For each component $M$, the equation sets are solved and the general formula is:

$$
I^{m}\left(\tau, \mu_{i}\right)=\sum_{j^{=-N}}^{N} L_{j}^{m} g_{j}^{m}\left(\mu_{i}\right) \exp \left(-k_{j}^{m}\right)+Z_{0}^{m}\left(\mu_{i}\right) \exp \left(-\tau / \mu_{0}\right)
$$

where $L_{j}^{m}$ are the constants of integration determined from the boundary conditions, $g_{j}^{m}\left(\mu_{i}\right)$ and $k_{j}^{m}$ are solutions of an algebraic eigen-value problem and $Z_{0}^{m}\left(\mu_{i}\right)$ is related to the source function. Thus, the total radiance is the sum of individual components through equation (10).

In the following analysis, flux $(F)$ is also used. The downward flux $\left(F_{d}\right)$ and 
upward flux $\left(F_{u}\right)$ for any given $\tau$ can be calculated

$$
\begin{aligned}
F_{u}(\tau) & =\int_{\partial}^{2 \pi} I(\tau, \mu, \phi) \mu d \mu d \phi \\
F_{d}(\tau) & =\iint_{\theta} I(\tau, \mu, \phi) \mu d \mu d \phi
\end{aligned}
$$

\section{Data analysis}

The remotely-sensed soil depth is determined by examining (i) the hemispheric transmittance profile in the soil $t(\tau)$; (ii) the sensitivity of hemispheric reflectance $r\left(\tau_{\text {air }}\right)$; and (iii) the sensitivity of bidirectional reflectance $R\left(\tau_{\mathrm{air}}, \mu, \phi\right)$. These quantities are defined below:

$$
\begin{aligned}
t(\tau) & =\frac{F_{d}(\tau)}{F_{d}\left(\tau_{\mathrm{air}}\right)} \\
r(\tau) & =\frac{F_{u}(\tau)}{F_{d}(\tau)} \\
R(\tau, \mu, \phi) & =\frac{I(\tau, \mu, \phi)}{\mu_{0} F_{0}}
\end{aligned}
$$

where $\tau_{\text {air }}$ is the total optical depth of the atmosphere.

To make computations simpler, only aerosol particles are considered in the atmosphere. The aerosol optical depth over the wavelength is assumed to follow

$$
\tau_{\mathrm{a}}=0.10 \lambda^{-1.1}
$$

and the single-scattering albedo is set to 0.94 . The phase function is equivalent to that of Haze L as specified by Garcia and Siewert (1985), and is shown in figure 1.

The first case is for a clay soil composed of spherical particles. The particle radius varies from 0 to $1 \mu \mathrm{m}$ with $r_{\text {eff }}=0.5 \mu \mathrm{m}$ and $v_{\text {eff }}=0.045 \mu \mathrm{m}$. The complex refractive indices are listed in table 1, which were measured from the Illite particles from Fithian, Illinois (Egan and Hilgeman 1979). Illite is a silicate belonging to the group of micas. Particles of micas are never spheroid. Treating them as spheroid is the first-degree approximation. The calculated extinction cross-section using Mie theory are also listed in the table. The longer the wavelength, the smaller the extinction cross-section.

Figure 2 shows the hemispheric transmittance at different wavelengths and different solar zenith angles. Transmittance in those figures has been normalized so that the top of the soil is set $\tau=0$. The hemispheric transmittance is obviously a strong function of the solar zenith angle and the wavelength. The attenuation rate is smaller with smaller solar zenith angles or longer wavelengths, but transmittance almost approaches zero at optical depth around $8-10$.

The geometric depth $z$ can be determined by

$$
z=\frac{\tau_{0} \frac{4}{3} \pi r_{\mathrm{eff}}^{3}}{\left\langle C_{\mathrm{ext}}\right\rangle f}
$$




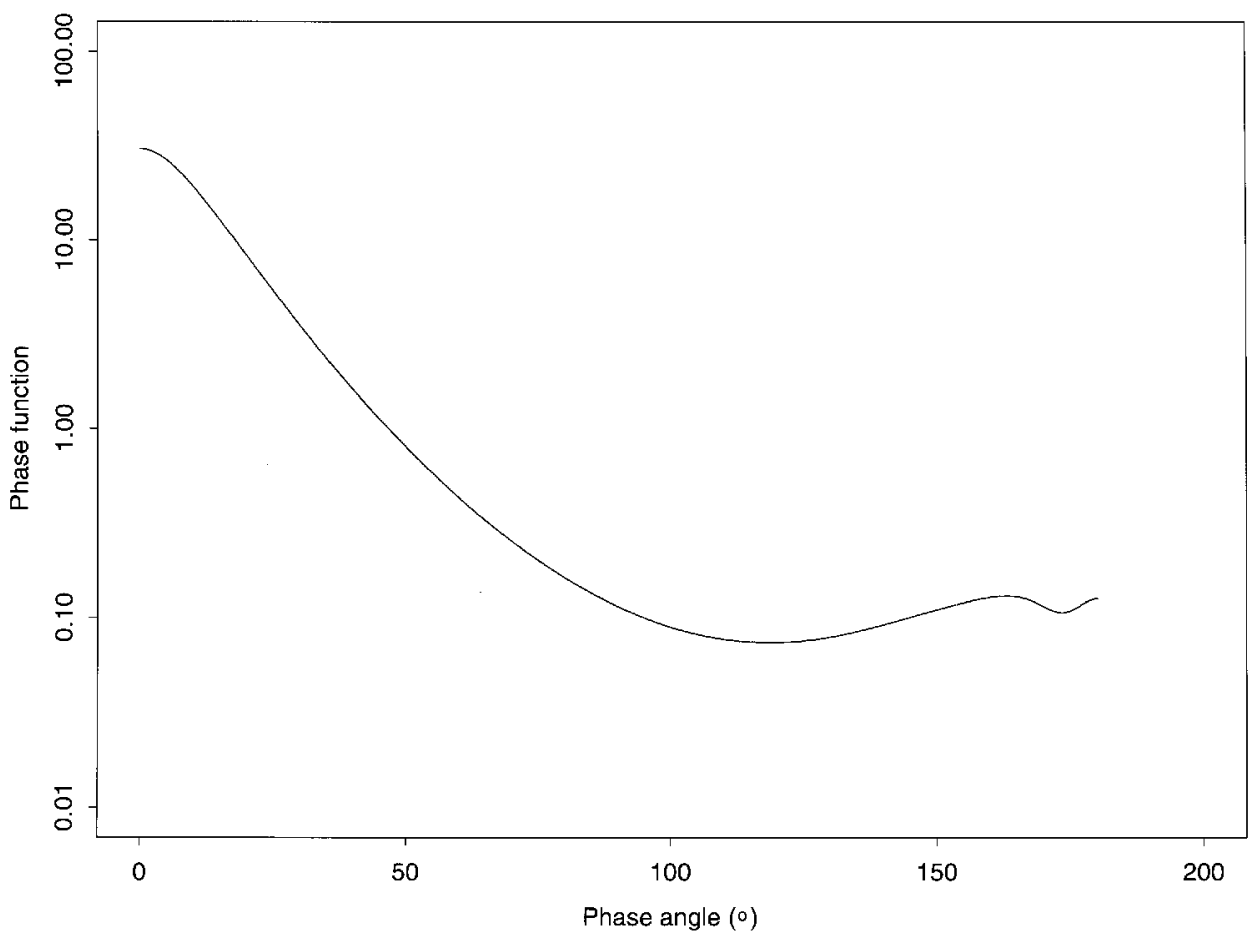

Figure 1. The phase function of the Haze $\mathrm{L}$ in the atmosphere.

Table 1. Soil refractive indices $\left(n_{r}+i n_{i}\right)$ and calculated extinction cross-section $C_{\text {ext }}$.

\begin{tabular}{|c|c|c|c|}
\hline Wavelength $(\mu \mathrm{m})$ & $n_{r}$ & $n_{i}$ & $C_{\mathrm{ext}}$ \\
\hline $0 \cdot 500$ & $1 \cdot 415$ & $0 \cdot 05029$ & $2 \cdot 3948$ \\
\hline $0 \cdot 600$ & $1 \cdot 411$ & $0 \cdot 04289$ & $2 \cdot 6143$ \\
\hline $0 \cdot 700$ & $1 \cdot 399$ & 0.05393 & $2 \cdot 5011$ \\
\hline $0 \cdot 817$ & $1 \cdot 395$ & $0 \cdot 05448$ & $2 \cdot 2960$ \\
\hline $0 \cdot 907$ & $1 \cdot 391$ & 0.05393 & $2 \cdot 0910$ \\
\hline $1 \cdot 000$ & $1 \cdot 387$ & $0 \cdot 05448$ & $1 \cdot 8705$ \\
\hline $1 \cdot 105$ & $1 \cdot 387$ & $0 \cdot 05340$ & $1 \cdot 6593$ \\
\hline
\end{tabular}

where $\tau_{0}$ is the so-called sensible optical depth. When the optical depth is larger than $\tau_{0}$, the indicators (bidirectional reflectance, transmittance, or directional albedo) will not vary significantly any more. In other words, the information beyond $\tau_{0}$ will not be detectable. If we assume the fraction volume occupied by soil particles $f=0 \cdot 3$, optical depth 10 corresponds to geometric depth $z=8 \mu \mathrm{m}$ at the wavelength $0.907 \mu \mathrm{m}$. If we applied equation (1) to determine the penetration depth, it roughly corresponds to transmittance $1 / e$. From those figures it can be seen that the optical depth with transmittance $1 / e$ is about $1 \cdot 5-3$. Thus, the geometric penetration depth is smaller.

Figure 2. Diffuse transmittance profiles of clay soils at different solar zenith angles and wavelengths: (a) compares diffuse transmittance of different solar zenith angles in both $500 \mathrm{~nm}$ and $907 \mathrm{~nm}$; $(b)$ compares diffuse transmittance of different wavelengths at both solar zenith angles $15^{\circ}$ and $55^{\circ}$. 


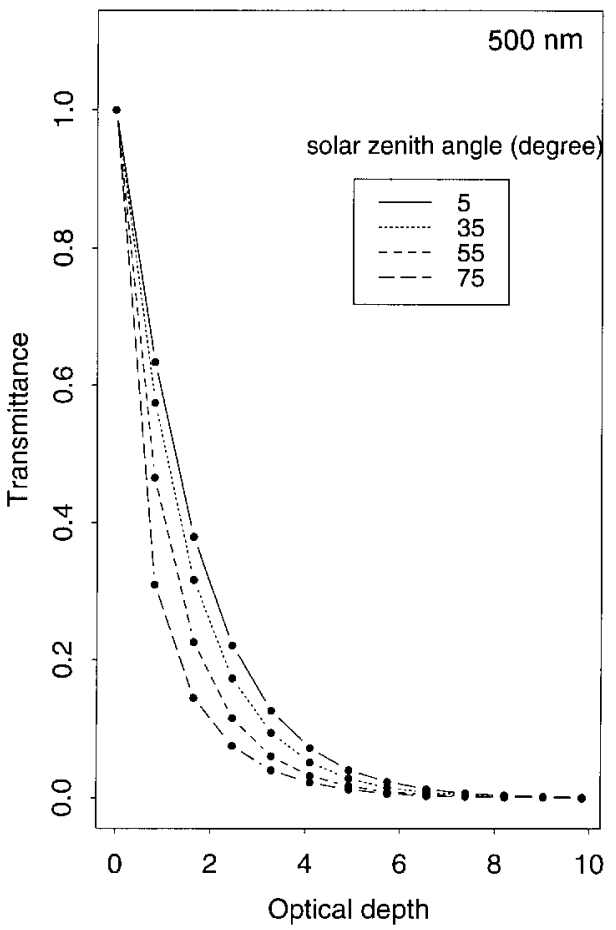

(a)

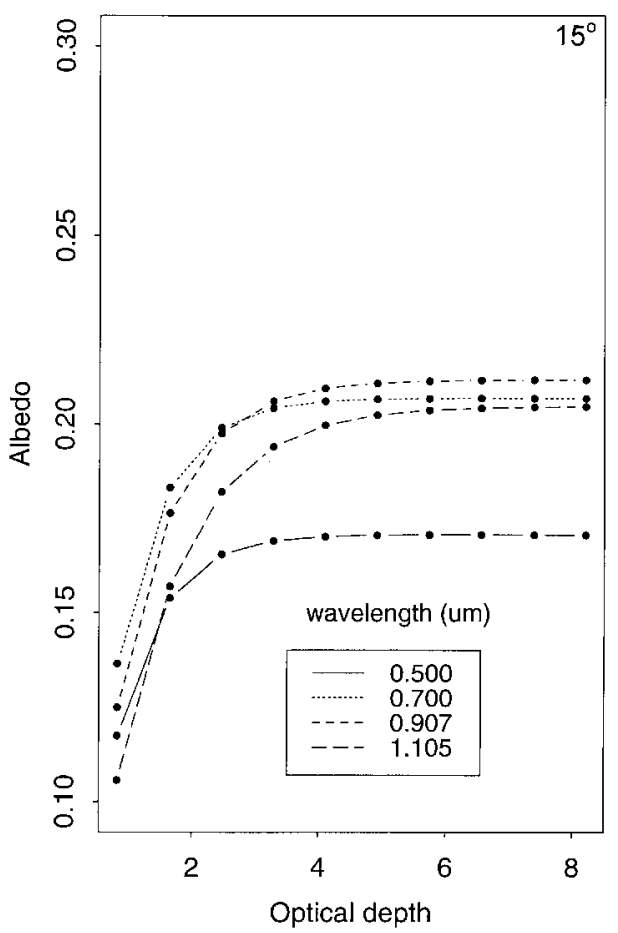

(c)

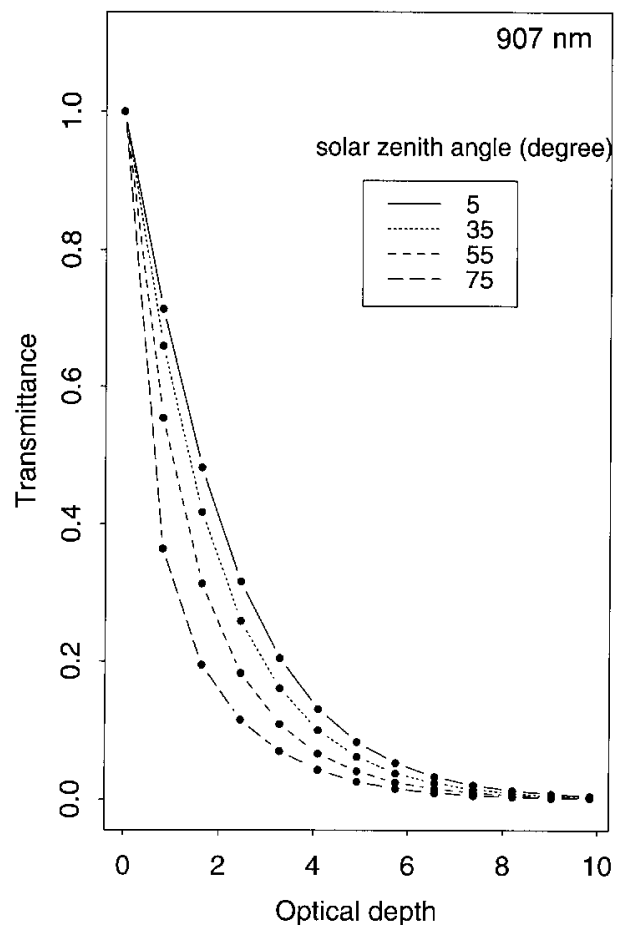

(b)

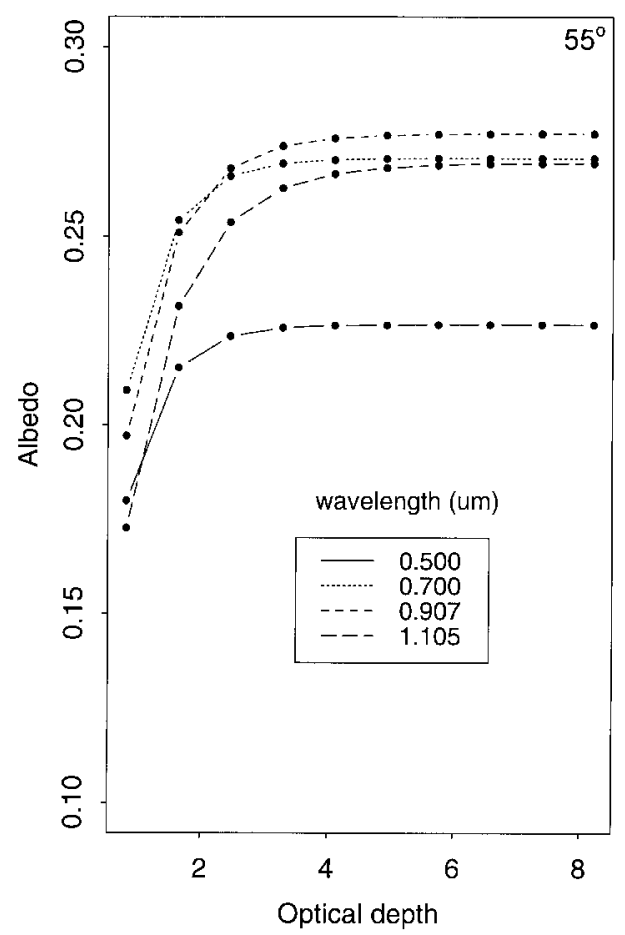

(d) 
We also calculated hemispherical reflectances by changing the optical depth. Figure 3 illustrates the computational results at different wavelengths and solar zenith angles. When the solar zenith angle is smaller, the calculated hemispheric reflectance is smaller, but the converged optical depth is slightly larger. Albedo at smaller wavelength is smaller but converged optical depth is larger. From this figure, we can see that the sensible optical depth is about 3-4. Since most sensors observe soil media at specific directions, thus, similar calculations are made for the bidirectional reflectance, rather than hemispheric reflectance. The results are presented in figure 4 . The sensible optical depth is very similar to that in the analysis of hemispheric reflectance, about 3-4. Optical depth 3 corresponds to geometric depth $2 \cdot 2 \mu \mathrm{n}$ at wavelength $0.5 \mu \mathrm{n}$ and $3.15 \mu \mathrm{n}$ at wavelength $1.105 \mu \mathrm{m}$.

All results discussed above came from calculations for clay particles. For larger soil particles, the sensible soil depth may be more easily predicted because the extinction efficiency $Q_{\text {ext }}$ that can be used to calculate the extinction coefficient asymptotically approaches 2 :

$$
\left\langle C_{\mathrm{ext}}\right\rangle=Q_{\mathrm{ext}} \pi r_{\mathrm{eff}}^{2}
$$

Thus, equation (17) can be simplified as

$$
z \approx \frac{2 \tau_{0} t_{\mathrm{eff}}}{3 f}
$$

In order to determine the sensible optical depth $\tau_{0}$, we need to solve the radiative transfer equation several times for different total optical depths of the soil medium. The sensible optical depth corresponds to that where the reflectance or other indicators will not change significantly as the total optical depth increases. The basic procedure of solving a radiative transfer equation has been described earlier. However, appropriate scaling is required for the medium composed of large particles. When the particle size increases, the forward scattering peak in the phase function becomes more dominant, which may result in high numerical uncertainty. An example is displayed in figure 5 , where phase functions of clay, silt and sand soils are calculated by the Mie code in wavelength $\lambda=0.817 \mu \mathrm{m}$. The complex refractive indices for clay and silt are listed in table 1 , and the value for sand is $1.555+i 0.0147$, which was measured from Bytownite samples in Minnesota (Egan and Hilgeman 1979). From the figure, we can see that the phase function of the sand has a very sharp peak. The scaling processing is straightforward. The phase function is expanded in a series of Legendre polynomials:

$$
P(\theta)=\sum_{n=0}^{M^{-1}}(2 n+1) \alpha_{n} p_{n}(\cos (\theta))
$$

where $M$ is the number of terms in the series. For a highly anisotropic phase function, $M$ has to be very large. The scaling process is to retain much smaller number of

Figure 3. Dependence of the hemispheric reflectance of clay soils on soil optical depth at different solar zenith angles and wavelengths: $(a)$ compares hemispheric reflectance of different solar zenith angles in both $500 \mathrm{~nm}$ and $907 \mathrm{~nm}$; $(b)$ compares hemispheric reflectance of different wavelengths at both solar zenith angles $15^{\circ}$ and $55^{\circ}$. 


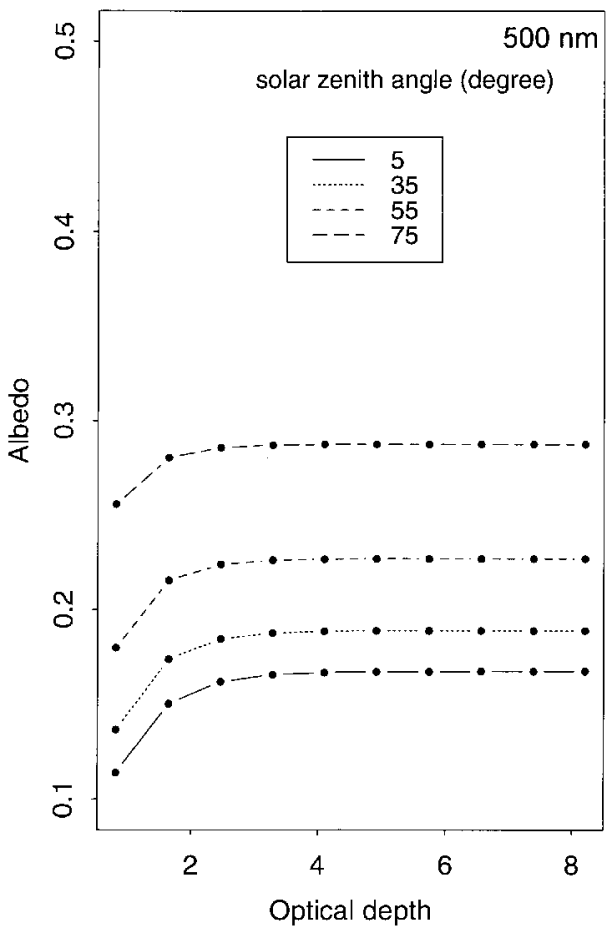

(a)

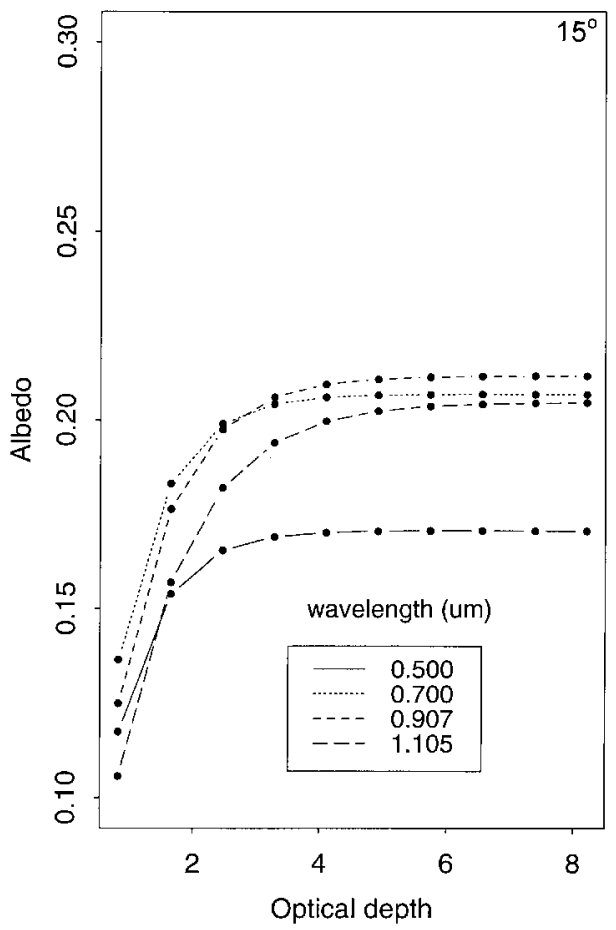

(c)

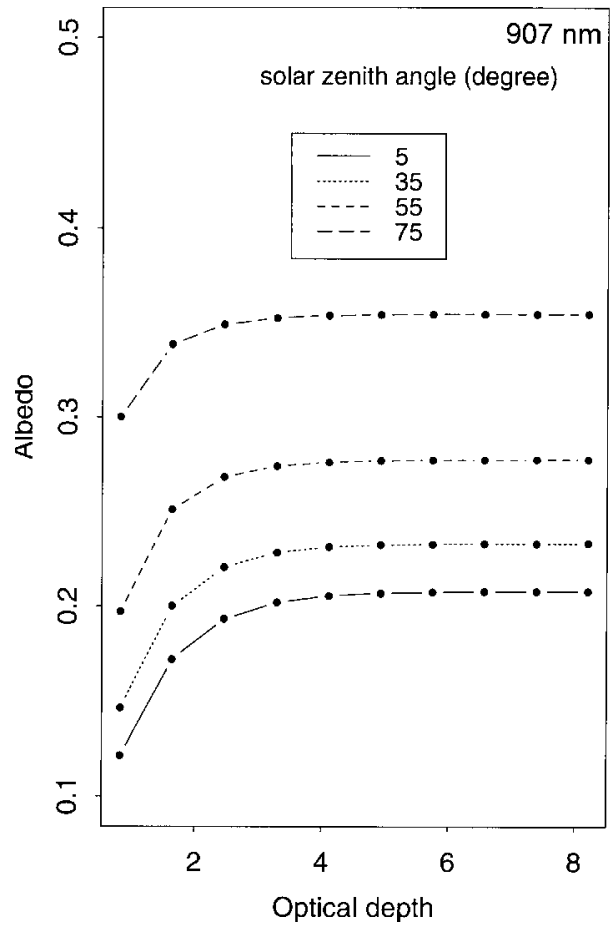

(b)

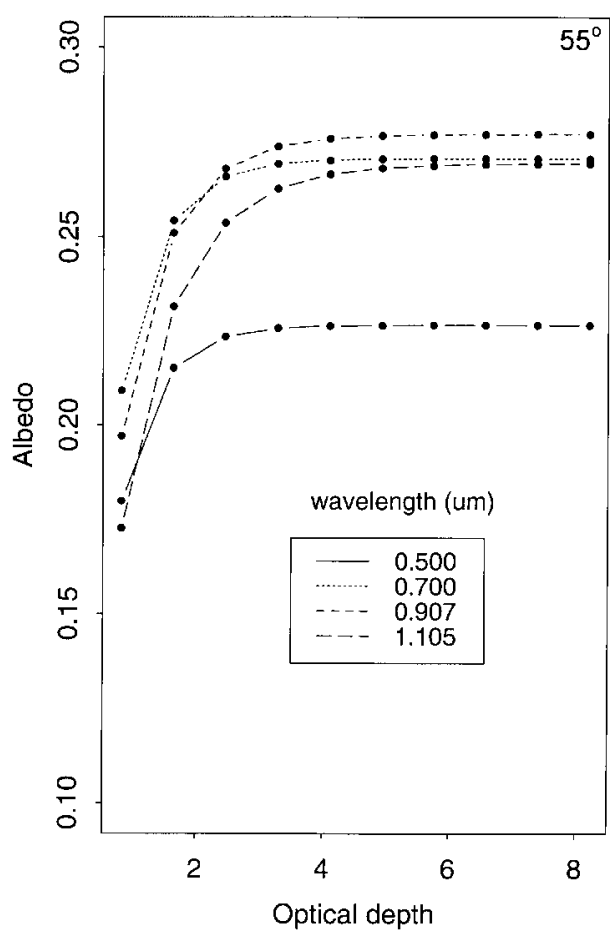

(d) 


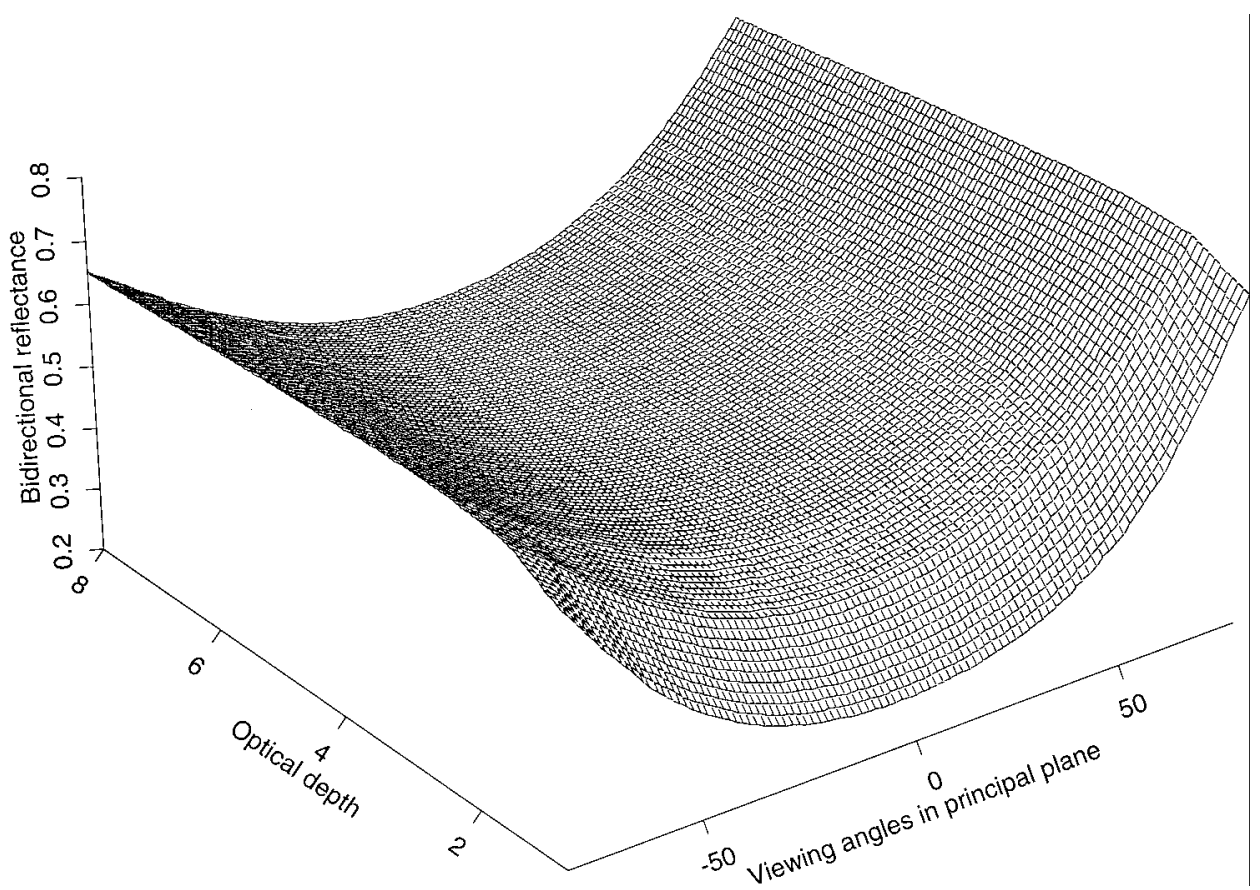

(a)

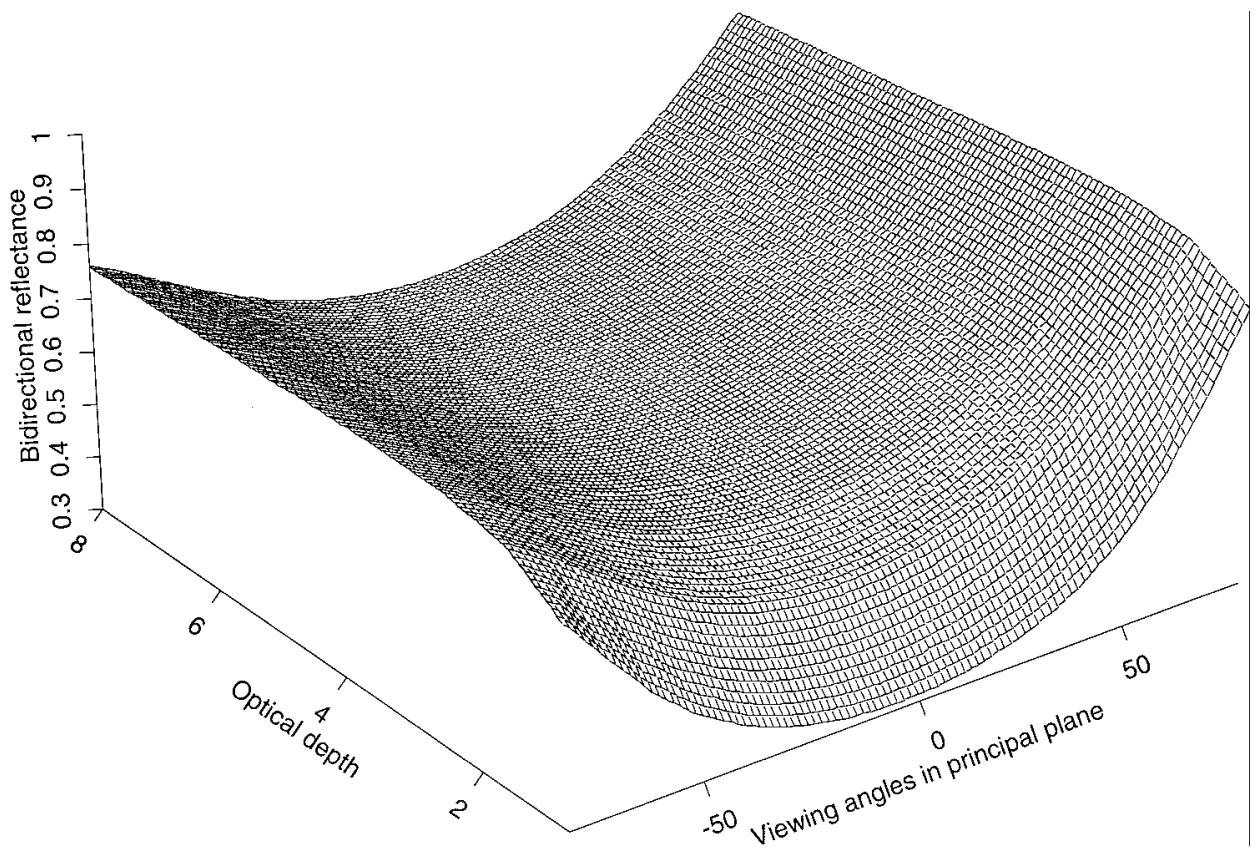

(b)

Figure 4. Dependence of the bidirectional reflectance of clay soils in the solar principal plane in two wavelengths $(a) 500 \mathrm{~nm}$ and $(b) 700 \mathrm{~nm}$. 


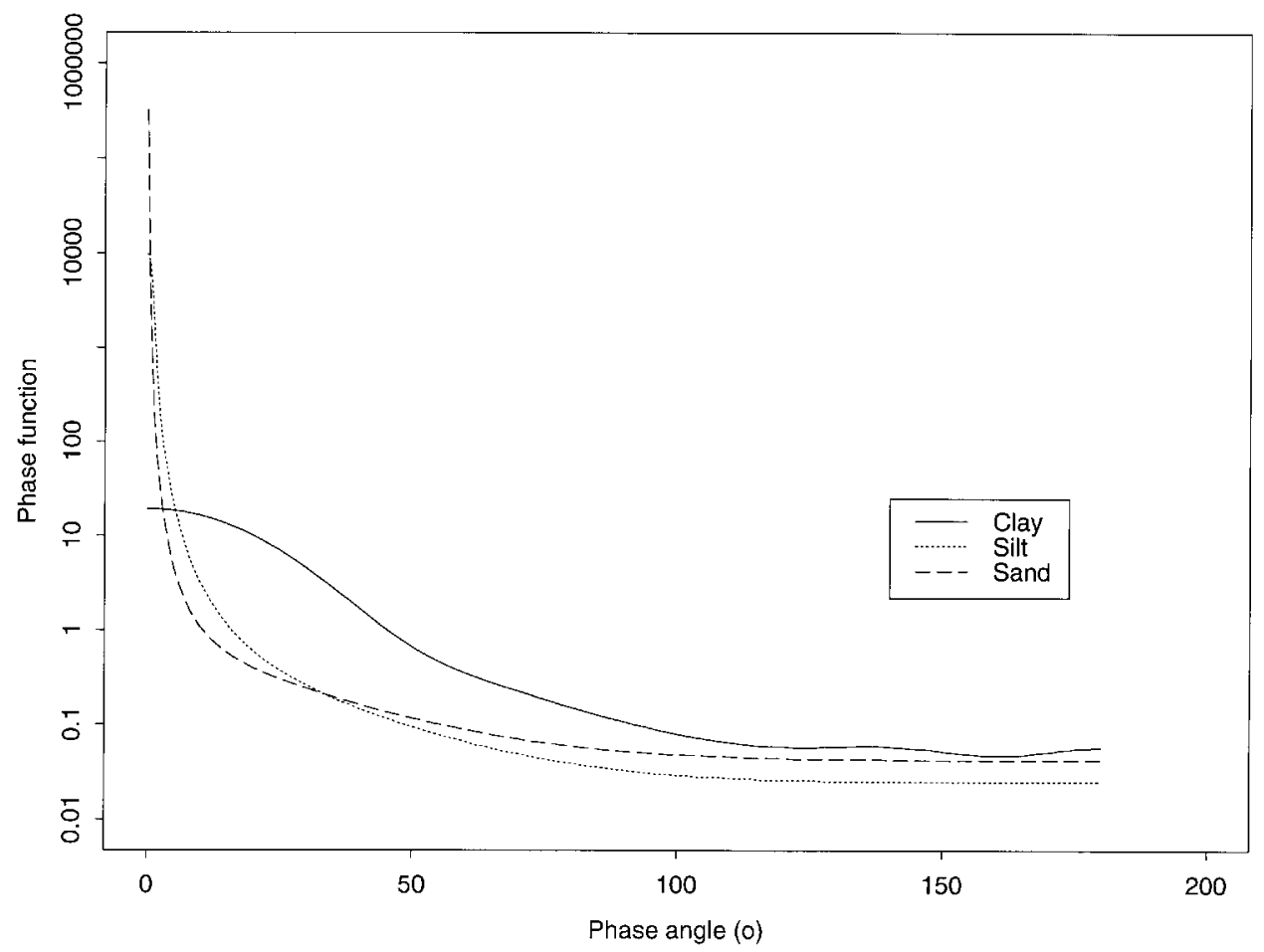

Figure 5. Phase functions of clay, silt, and sand soils.

terms, say, $M_{1} \ll M$, by modifying the expansion coefficients but keeping the same expansion formula. The scaled coefficient $b_{n}$ is related to the original coefficients $\alpha_{n}$

$$
b_{n}=\frac{\alpha_{n}-f}{1-f}
$$

where the forward fraction parameter $f$ can be defined as

$$
f=\alpha_{M_{1}}
$$

In order to produce the same results by radiative transfer calculations, the singlescattering albedo $(\omega)$ and optical depth $(\tau)$ have to be scaled as well:

$$
\begin{aligned}
\tau^{\prime} & =(1-\omega f) \tau \\
\omega^{\prime} & =\frac{(1-f) \omega}{(1-\omega f)}
\end{aligned}
$$

In order to avoid negative values of the phase function, Kim and Lee (1990) suggested to further modify the scaled phased function by adding a positive constant. More correction procedures were discussed by Nakajima and Tanaka (1988) to achieve a high accuracy.

Figure 6 presents the dependence of hemispheric reflectance on optical depth at solar zenith angles $5^{\circ}$ and $65^{\circ}$. The sensible optical depth of clay soils is larger than 3 , but the sensible optical depths of silt and sand are about 2-3 under these two 


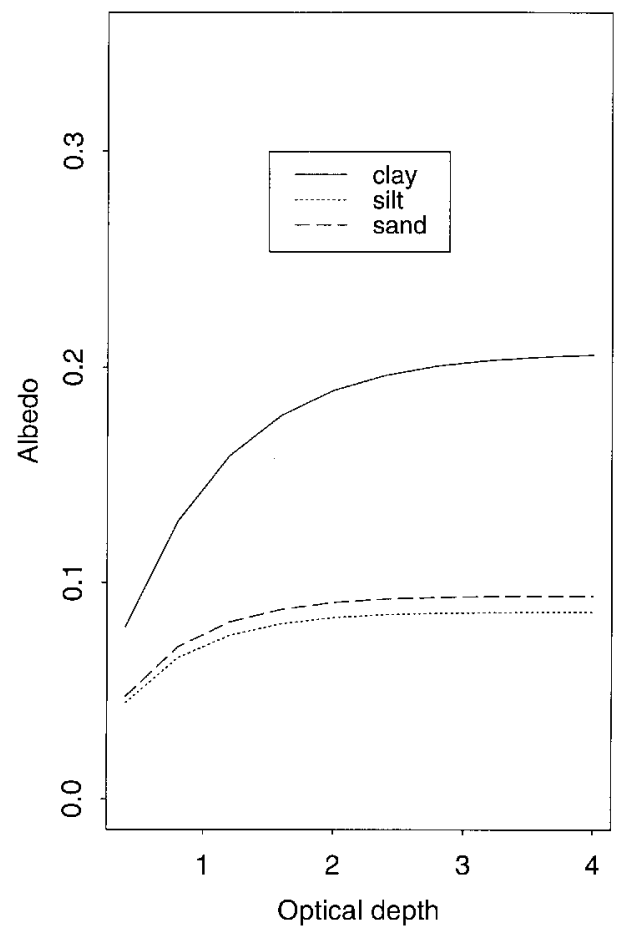

(a)

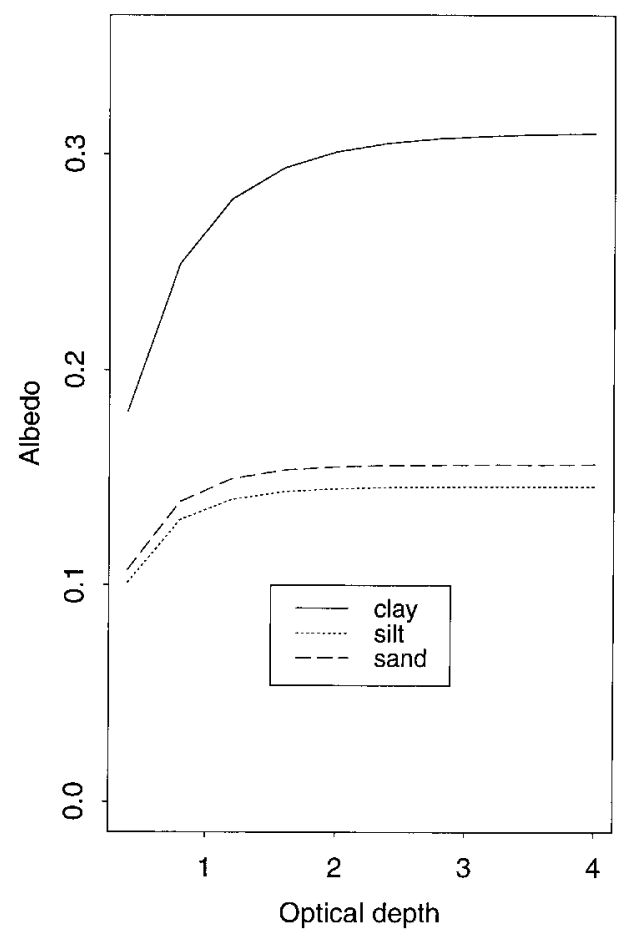

(b)

Figure 6. Dependence of hemispheric reflectance of different particle sizes on optical depth at solar zenith angles $(a) 5^{\circ}$ and $(b) 65^{\circ}$.

illumination conditions. If $\tau_{0}=3$ and let $f=0 \cdot 5$, the sensible geometric depth soils composed of large particles will be about $4 r_{\text {eff. }}$.

Another experiment was designed to examine the effects of particle shapes. Particles are assumed spheroidal, the ratio of the major and minor axes are 0.5 and 2.0. The particles follow gamma size distribution, but two ranges are calculated separately. The calculated hemispheric reflectances are shown in figure 7. Particles of different shapes have variable hemispheric reflectances, but the sensible optical depths are almost the same. It is probably due to the fact that soil particles have very small transmittance and the volume density is one of the most significant factors controlling the sensible depth.

\section{Conclusions}

A number of numerical calculations are carried out to estimate the sensible depth of soils in the visible and near-IR spectral region. The atmosphere and soil are considered as a coupled medium. Aerosol is assumed to be Haze-L in the atmosphere. Soil particles are assumed spherical or ellipsoidal following different size distributions. Mie theory and $T$-matrix theory are used to calculate optical properties of spherical and ellipsoidal particles respectively. Three quantities are evaluated to find out the sensible depth: downward transmittance, hemispheric reflectance and bidirectional reflectance. Overall, the sensible optical depth is about 3 on average, which corresponds to the geometric depth 4 times the effective particle radius. The sensible soil depth is larger at longer wavelengths or smaller solar zenith angles. 


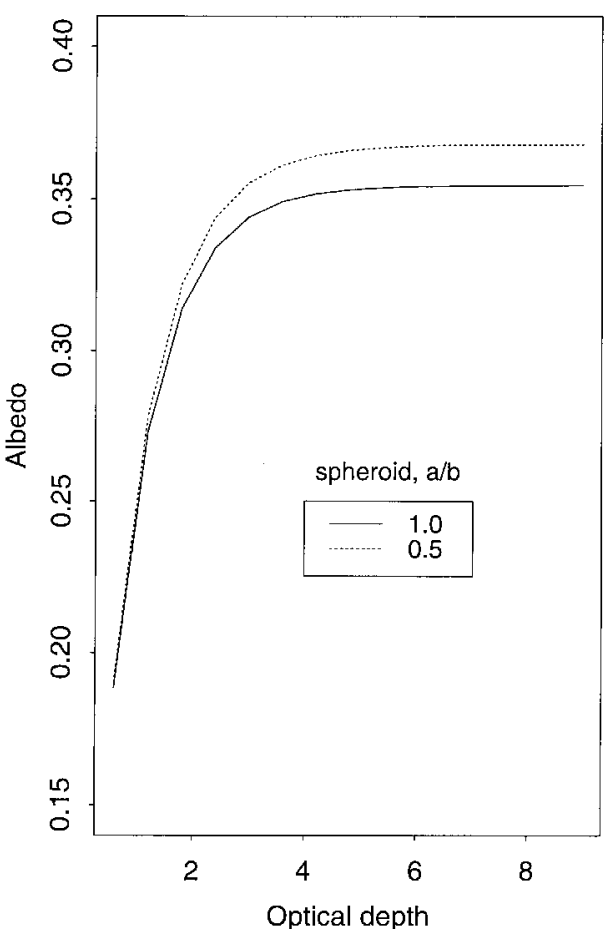

(a)

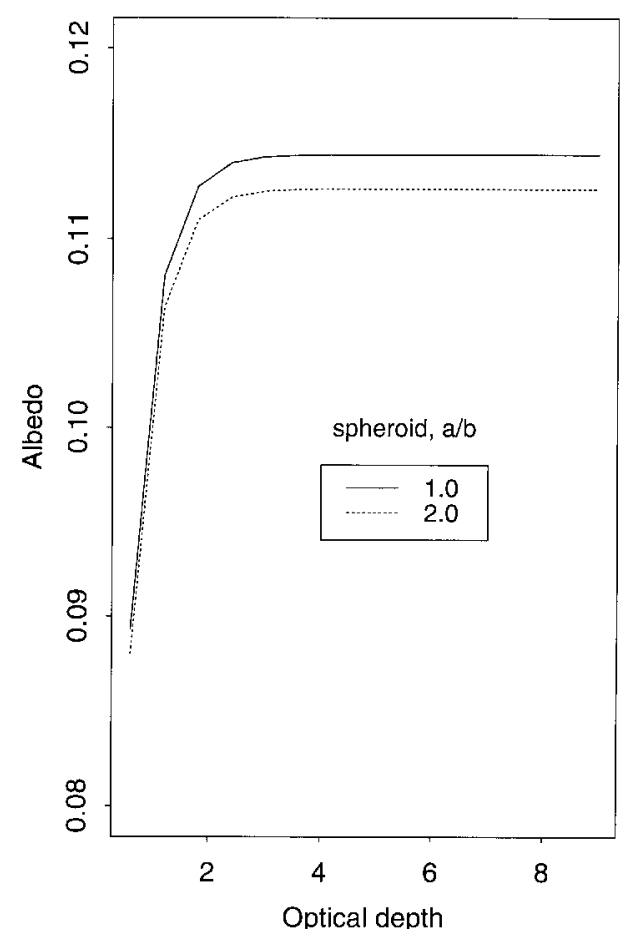

(b)

Figure 7. Dependence of hemispheric reflectance of different particle shapes on optical depth at solar zenith angle $45^{\circ}$. (a) coarse, (b) fine.

For soils composed of large particles, such as sandy soil, the sensible depth is relatively small. In such a case, radiative transfer modeling may not be a best choice since the soil surface roughness and mutual shadowing of neighboring particles may become the dominant factors. New models based on the geometric-optical principles may be more suitable. Based on numerical calculations, the verticle profiles of the soil moisture, temperature, organic matter, etc. do not seem necessarily important in the visible and near-IR spectral regions, which may simplify modeling significantly.

\section{Acknowledgements}

The author is grateful for Dr Michael Mishchenko for providing his $T$-matrix codes and valuable discussions, and David Shirey for his assistance in the manuscript preparation.

\section{References}

Ahmad, S. P., and Deering, D. W., 1993, A simple analytic function for bidirectional reflectance. Journal of Geophysical Research, 97, 18867-18886.

Bohren, C. F., and Huffman, D. R., 1983, Absorption and Scattering of Light by Small Particles (New York: John Wiley).

Buchan, G. D., 1989, Applicability of the simple lognormal model to particle-size distribution in soils. Soil Science, 147, 155-161.

Dapples, E. C., 1975, Laws of distribution applied to sand sizes. Geological Society of American Memoir, 142, 37-61.

Egan, W. G., and Hilgeman, T. W., 1979, Optical Properties of Inhomogeneous Materials: 
Applications to Geology, Astronomy, Chemistry, and Engineering (New York: Academic Press).

Garcia, R. D. M., and Siewert, C. E., 1985, Benchmark results in radiative transfer. Transport Theory and Statistical Physics, 14, 437-483.

Hansen, J. E., and Travis, L. D., 1974, Light scattering in planetary atmospheres. Space Science Reviews, 16, 527-610.

Hapke, B. W., 1981, Bidirectional reflectance spectroscopy 1. Theory. Journal of Geophysical Research, 86, 3039-3054.

HAPKe, B., 1993, Theory of reflectance and emittance spectroscopy (Cambridge: Cambridge University Press).

Irons, J. R., Weismiller, R. A., and Petersen, G. W., 1989, Soil reflectance. In Theory and Applications of Optical Remote Sensing, edited by G. Asrar (New York: John Wiley), pp. 66-106.

Jacquemoud, S., Baret, F., and HanocQ, J. F., 1992, Modelling spectral and bidirectional soil reflectance. Remote Sensing of Environment, 41, 123-132.

KIM, T. K., and LEE, H. S., 1990, Modified $\delta M$ scaling results for Mie-anisotropic scattering media. Journal of Heat Transfer, 112, 988-994.

Lenoble, J., 1985, Radiative Transfer in Scattering and Absorbing Atmospheres: Standard Computational Procedures (Hampton, Virginia Deepak).

Liang, S., and STRAHLER, A. H., 1994, Four-stream solution for atmospheric radiative transfer over an non-Lambertian surface. Applied Optics, 33, 5745-5753.

Liang, S., and Townshend, J. R. G., 1996a, A modified Hapke model for soil bidirectional reflectance. Remote Sensing of Environment, 55, 1-10.

LIANG, S., and TownSHEND, J. R. G., 1996b, A parametric soil BRDF model: a four stream approximation for multiple scattering. International Journal of Remote Sensing, 17, $1303-1315$.

Liang, S., and Mishchenko, M. I., 1997, Calculation of the soil hot spot effects using the coherent backscattering theory. Remote Sensing of Environment, 60, 163-173.

Lindner, B. L., 1988, Ozone on Mars: the effects of clouds and airborne dust. Planetary and Space Science, 36, 125-144.

Lummerzheim, D., Rees, W. R., and Anderson, H. R., 1989, Angular dependent transport of auroral electrons in the upper atmosphere. Planetary and Space Science, 37, 109-129.

Mishchenko, M. I., 1993, Light scattering by size-shape distributions of randomly oriented axially symmetric particles of a size comparable to a wavelength. Applied Optics, 32, 4652-4666.

MishchenKo, M. I., 1994, Asymmetry parameters of the phase function for densely packed scattering grains. Journal of Quantitative Spectroscopy and Radiative Transfer, 52, $95-110$.

Nakajima, T., and Tanaka, M., 1988, Algorithms for radiative intensity calculations in moderately thick atmospheres using a truncation approximation. Journal of Quantitative Spectroscopy and Radiative Transfer, 40, 51-69.

Pinty, B., Verstraete, M. M., and Dickson, R. E., 1989, A physical model for predicting bidirectional reflectance over bare soil. Remote Sensing of Environment, 27, 273-288.

Privette, J. L., Myneni, R. B., Emery, W. J., and Pinty, B., 1995, Inversion of a soil bidirectional reflectance model for use with vegetation reflectance models. Journal of Geophysical Research, 100, 25497-25508.

Stamnes, K., Tsay, S. C., Wiscombe, W., and Jayaweera, K., 1988, Numerically stable algorithm for discrete-ordinate-method radiative transfer in multiple scattering and emitting layered media. Applied Optics, 27, 2502-2509.

Stamnes, K., Tsay, S. C., Wiscombe, W., 1996, A general-purpose, numerically stable computer code for discrete-ordinate-method radiative transfer in scattering and emitting layered media: documentation of methodology. Unpublished paper.

Tsay, S. C., Stamnes, K., and JayaweEra, K., 1989, Radiative energy budget in the cloudy and hazy arctic. Journal of Atmospheric Sciences, 46, 1002-1018.

Tsay, S. C., and Stamnes, K., 1992, Ultraviolet radiation in the Arctic: the impact of potential ozone depletions and cloud effects. Journal of Geophysical Research, 97, 7829-7840.

Ulaby, F. T., Moore, R. K., and Fung, A. K., 1982, Microwave Remote Sensing: Active and Passive. II: Radar Remote Sensing and Surface Scattering and Emission Theory (Reading, Massachusetts: Addison-Wesley). 